\title{
On a sufficient optimality condition over convex feasible regions
}

\section{C.D. Alders and V.A. Sposito}

In this note a sufficient optimality condition is established for nonlinear programming problems over arbitrary cone domains. A Kuhn-Tucker type sufficient condition is established if the programing problem has a pseudoconvex objective function and a convex feasible region.

\section{Introduction}

Recent articles in the literature, [1], [2], [3], [4], [6], and [8] have established various sufficient optimality theorems. These extensions have involved replacing orthant domains by cone domains and sometimes allowing the constraints to be quasiconvex. This note establishes a KuhnTucker type sufficient condition, [5] and [7], for programming problems with a pseudoconvex objective function and a convex feasible region.

\section{Definitions}

ASSUMPTION 1. $P$ is an open set in $E^{n} \cdot \theta$ is a numerical function, $g$ is an m-dimensional vector function, and $h$ is a $k$-dimensional vector function, each defined on $P$. Also $\theta, g$, and $h$ are differentiable at $\bar{x}$.

DEFINITION 1. $C$ will denote any arbitrary cone in $E^{m}$.

DEFINITION 2. $C^{*}$ will denote the polar cone of $C$; that is, $C^{*}=\left\{y^{*} \in E^{m}: y^{*} y \geq 0\right.$ for all $\left.y \in C\right\}$.

Received 29 October 1976. 
DEFINITION 3 (Pseudoconvex). Let $\theta$ be a numerical function defined on an open set $P \subset E^{n}$ and let $Q_{1}^{-}$denote the negative orthant in $E^{I} \cdot \theta$ is pseudoconvex at $\bar{x}$ with respect to $Q_{I}^{-}$on $P$ if $\theta$ is differentiable at $\bar{x}$ and

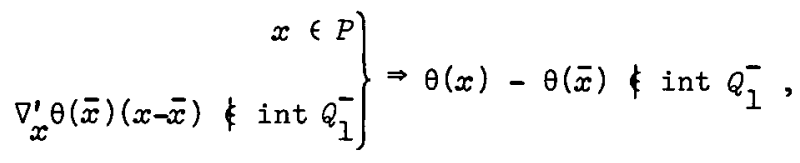

or, equivalently,

$$
\left.\begin{array}{r}
x \in P \\
\theta(x)-\theta(\bar{x}) \in \text { int } Q_{1}^{-}
\end{array}\right\} \Rightarrow \nabla_{x}^{\prime} \theta(\bar{x})(x-\bar{x}) \in \operatorname{int} Q_{1}^{-} .
$$

DEFINITION 4 (Minimization problem). The minimization problem is to find $\bar{x} \in E^{n}$, if it exists, such that

$$
\begin{aligned}
\theta(\bar{x}) & =\min \theta(x), \\
\bar{x} & \in X,
\end{aligned}
$$

where

$$
X=\left\{x: x \in P \subset E^{n}, g(x) \in C \subset E^{m}, h(x)=\{0\} \subset E^{k}\right\} .
$$

DEFINITION 5 (Kuhn-Tucker problem) (see [5], pp. 94, 162-163). The following is a modified Kuhn-Tucker stationary point problem over cone domains.

Find an $\bar{x} \in P \subset E^{n}, \bar{r} \in-C^{*} \subset E^{m}$, and $\bar{s} \in E^{k}$ such that

$$
\begin{gathered}
\nabla_{x}^{\prime} \theta(\bar{x})+\bar{r}^{\prime} \nabla_{x} g(\bar{x})+\bar{s}^{\prime} \nabla_{x} h(\bar{x})=0, \\
\bar{r}^{\prime} g(\bar{x})=0, \\
g(\bar{x}) \in C, \\
h(\bar{x})=\{0\}, \\
(\bar{x}, \bar{s}) \in-C^{*} x E^{k} .
\end{gathered}
$$




\section{Sufficient optimality condition}

LEMMA 1 . Let $X$ be a convex set contained in an open set $P$ in $E^{n}$. Let $f$ be a numerical function defined on $P$ and differentiable at $\bar{x}$. Also let $f(\bar{x})=0$, where $\bar{x} \in X$. If $f(x)>0$ has no solution $x \in X$, then $\nabla^{\prime} f(\bar{x})(x-\bar{x})>0$ has no solution $x \in X$.

Proof. We will prove the contrapositive, that is, if $\nabla^{\prime} f(\bar{x})(x-\bar{x})>0$ has a solution $\hat{x} \in X$, then $f(x)>0$ has a solution $\tilde{x} \in X$.

Since $X$ is convex, $\bar{x}+t(\hat{x}-\bar{x}) \in X$, for some $t \in(0,1)$ and since $f$ is differentiable at $\bar{x}$, we have that

$$
f(\bar{x}+t(\hat{x}-\bar{x}))=f(\bar{x})+t \nabla^{\prime} f(\bar{x})(\hat{x}-\bar{x})+o(t) .
$$

Now with $f(\bar{x})=0$ and $\nabla^{\prime} f(\bar{x})(\hat{x}-\bar{x})>0$ we have, for sufficiently small $t, f(\bar{x}+t(\hat{x}-\bar{x}))>0$. So letting $\tilde{x}=\bar{x}+t(\hat{x}-\bar{x})$, the result follows. The case where $X=\{\bar{x}\}$ follows immediately.

THEOREM 1. Let $P, \theta, g$, and $h$ satisfy Assumption 1 with $\theta$ pseudoconvex with respect to $\bar{Q}_{1}$ at $\bar{x}$ on $P ; C$ be an arbitrary cone; and assume that the feasible region $X$ of the minimization problem is convex. If there exists a solution to the Kuhn-Tucker problem, then $\bar{x}$ is a solution to the minimization problem.

Proof. Assume there exists a solution to the Kuhn-Tucker problem. Since $X$ is convex, consider Lemma 1 with $f(x)=\bar{r}^{\prime} g(x)+\bar{s}^{\prime} h(x)$ where $\bar{r} \in-C^{*}, g(x) \in C$, and $h(x)=\{0\}$ for any $x \in X$. Now if there exists a solution to the Kuhn-Tucker problem, then

$$
\left[\nabla^{\prime} \theta(\bar{x})+\bar{x}^{\prime} \nabla g(\bar{x})+\bar{s}^{\prime} \nabla h(x)\right](x-\bar{x})=0 \text { for all } x \in X \text {. }
$$

Now appealing to Lemma 1 , we have that

$$
\left[\bar{r}^{\prime} \nabla g(\bar{x})+\bar{s}^{\prime} \nabla h(\bar{x})\right](x-\bar{x}) \leq 0 \text { for all } x \in X \text {. }
$$

Hence

$$
\nabla^{\prime} \theta(\bar{x})(x-\bar{x}) \geq 0 \text { for all } x \in X \text {, }
$$

and since $\theta$ is pseudoconvex, it follows that $\bar{x}$ is optimal. 


\section{References}

[1] J.M. Borwein, "A note on Fritz John sufficiency", Bulz. Austral. Math. Soc. 15 (1976), 293-296.

[2] B.D. Craven, "Sufficient Fritz John optimality conditions", BulZ. Austraz. Math. Soc. 13 (1975), 411-419.

[3] B.D. Craven and B. Mond, "A Fritz John theorem in complex space", Bul2. Austral. Math. Soc. 8 (1973), 215-220.

[4] T.R. Gulati, "A Fritz John type sufficient optimality theorem in complex space", Bull. Austral. Math. Soc. 11 (1974), 219-224.

[5] Olvi L. Mangasarian, Nonlineax programing (McGraw-Hill, New York, St. Louis, San Francisco, London, Sydney, Toronto, Mexico, 1969).

[6] V.A. Sposito, "Modified regularity conditions for nonlinear programming problems over mixed cone domains", Math. Programing 6 (1974), 167-179.

[7] V.A. Sposito, Linear and nonZinear programing (Iowa State University Press, Ames, 1975).

[8] Vince A. Sposito and H.T. David, "Saddle-point optimality criteria of nonlinear programming problems over cones without differentiability", SIAM J. Appl. Math. 20 (1971), 698-702.

\footnotetext{
Department of Statistics,

lowa State University,

Ames,

lowa,

USA.
} 